\title{
Energy Dissipation in Spacecraft Structures Incorporating Bolted Joints with Viscoelastic Layers
}

\author{
R. Wang and A. D. Crocombe \\ University of Surrey, Guildford, Surrey, GU2 7XH, UK \\ G. Richardson \\ Surrey Space Technology Ltd., Guildford, Surrey, GU2 7XH, UK \\ and \\ C. I. Underwood \\ University of Surrey, Guildford, Surrey, GU2 7XH, UK
}

\begin{abstract}
The energy dissipation capacity of bolted joints with viscoelastic layers in a spacecraft structure was investigated. Initially a linear spring dashpot model was used to represent the bolts in a satellite structure. A relationship was developed between the model parameters (stiffness and damping coefficient) and the viscoelastic material and geometric properties (shear modulus, loss factor, operating area, and thickness) of the actual bolted joint. This model was then developed into the non-linear domain. Experiments on bolted joints with viscoelastic layers were carried out to provide information for the non-linear joint model. These models were incorporated into a simple spacecraft model to investigate the effect on the spacecraft response. Based on these numerical analysis, it was found the joints can dissipate much energy and the response of the spacecraft structure to vibrations during launch can be decreased significantly.
\end{abstract}

\section{Introduction}

Surrey Satellite Technology Limited (SSTL) developed a new spacecraft design, composed of honeycomb panels connected by many bolted joints. The energy dissipation capacity of those joints was of interest to the designers. Former research [1] showed that the damping capacity was quite low for plain joints operating in the micro-slip range. By using Finite Element analaysis, it was found that the percentage of the energy dissipated in the satellite deriving from the joints was not over 5\% when the structural damping coefficient was 0.04 and the excitation acceleration was $2 \mathrm{~g}$, an acceleration used to characterise the launch of this satellite.

Ways of increasing the energy dissipation capacity of bolted joints in the spacecraft, thus decreasing the structural response, have been investigated and an approach incorporating viscoelastic layers in the bolted joints is presented here. Viscoelastic dampers have long been used in the control of vibration and noise in aerospace structures, engine mounts and industrial machines as well as large civil engineering structures. It is well known that 
the behaviour of viscoelastic materials is both strain and strain rate dependent whilst also depending on temperature. A widely used model for viscoelastic materials involves specifying a complex modulus [2]. Both the real and the imaginary parts change with frequency and temperature. This model forms a good approximation in many situations. However, when the strain is large and more accuracy is required, more complicated models are needed to deal with the non-linearity. The simpler type of models, which do not consider strain dependency have been termed linear models in this paper. Those models that include both strain and strain rate dependency, have been termed non-linear models.

In the linear domain, much research has been done. For example, Johnson [3] presented three different ways of solving the equations of motion for structures with a viscoelastic material: a) the complex eigenvalue method; b) the modal strain energy method (SEM); and c) the direct frequency response method. These methods are quite commonly used in Finite Element software. Method c) has been used in this paper for a detailed Finite Element analysis of an individual bolted joint with linear viscoelastic layer in Nastran. The viscoelastic material was represented by frequency dependent storage and loss shear moduli.

When analyzing a structure with many bolted joints it is not feasible to model the joints in such detail and a simpler representation for their time dependent response is required. There are many "simple" analytical models that can be used to model a linear visco-elastic response. The most common ones are spring dashpot systems, for example, Maxwell and Kelvin models. In the Maxwell model a spring and a dashpot are used in series. In the Kelvin model they are used in parallel. These two models are the simplest analytical forms for a viscoelastic material. Many other analytical models are different combinations of these two models. These kinds of models have been called standard mechanical models (SMM) in Park's paper [5] and have been found to be quite efficient. Other common linear models include the Zener model [6] and Havriliak-Negami model [7] provide a better frequency representation but at the cost of additional model parameters. In the structural modelling reported in this paper the simpler models have been shown to be entirely appropriate and the simpler formulation forms a good foundation for the non-linear development undertaken.

Considering the non-linear domain a common approach [eg 8, 9] is based on a form of the convolution integral with non-linear material descriptors. Kitagawa et al. [10] gave a review of literature that focused on defining a 
constitutive law in non-linear stress and strain space using the concept of overstress, where the rate dependency was dependent of the deviation form an equilibrium non-linear material response. Bergstrom et al. [11] extends this overstress concept to networks, an equilibrium network corresponding to the state that is approached in long term stress relaxation tests and a second network capturing the non-linear rate-dependent deviation from the equilibrium state. Many experiments have been carried out to validate these non-linear visco-elastic models. Such work provides a good understanding of the non-linear response of visco-elastic materials. However, although they have been found to provide a good representation of selected material data, extensive calibration is required to determine the model

parameters. Furthermore, when modelling a structure with many bolted joints a much simpler, non-linear representation of the joints is required. In the work reported here this has been achieved by a novel non-linear extension of the linear spring-dashpot models discussed in the previous paragraph.

In the next two sections, methods of incorporating a linear and non-linear viscoelastic layered bolted joint model into a spacecraft structural model will be discussed. The effects of the joints on the response of the spacecraft structure will be shown.

\section{Energy dissipated in a spacecraft structure incorporating linear viscoelastic layered}

\section{bolted joints}

The work reported in this section focuses on linear visco-elastic models. Initially a simplified analytical model governing the behaviour of a bolted joint unit are developed and compared with more accurate FE models. This validated simplified analytical model is then used as the basis for developing spring-dashpot representations of the bolted joint unit. Finally these spring dashpot models are used to represent bolted joints in a satellite model and estimates of increased damping levels obtained.

\section{A. Modeling of bolted joints with a viscoelastic layer}

1. Viscoelastic material properties 
A viscoelastic material has both elastic and viscous properties. So, under dynamic loads there is a phase difference between the excitation load and the displacement response. Normally this is represented by a complex modulus $G^{*}=(1+\mathrm{i} \eta) G$. It has been found that the shear modulus $G$ and the loss factor $\eta$ depend on frequency as well as temperature. Much research has been undertaken on different materials by other researchers. Here Stahle's [12] experiment data for SMRD 100F90, shown in Fig. 1, will be used for the viscoelastic layers in bolted joints because this material has been used in spacecraft applications already. The values in Table 1 were obtained from Fig. 1 at a temperature $25^{\circ} \mathrm{C}$. This temperature is chosen because the focus of this research is on the launch situation

\section{Estimation of the energy dissipated in a viscoelastic layer in shear}

In order to estimate the energy dissipation capability of a viscoelastic material in shear, a simple calculation has been introduced. The harmonic shear force $F$ and the corresponding displacement $x$ acting on the element of material with shear area of $A$ and transverse thickness of $h$ are shown in Fig. 2. This force and displacement are linked, via the shear stress and strain through the complex shear modulus as

$$
\frac{F}{A}=(1+i \eta) G \frac{x}{h}
$$

For harmonic loading, where $F=F_{\mathrm{o}} \cos \omega t$, Eqn 1 can be solved to give the harmonic displacement $x$ as

$$
x=x_{o} \cos (\omega t-\theta)
$$

where

$$
x_{o}=\frac{F_{o} h}{A G \sqrt{1+\eta^{2}}} ; \quad \sin \theta=\frac{\eta}{\sqrt{1+\eta^{2}}}
$$

The energy dissipated $E$ can be found as the area defined by the force-displacement hysteresis loop . This is given below and the harmonic displacement $x$ can be substituted to give

$$
E=\pi F x \sin \theta=\frac{\pi F^{2} h \eta}{A G\left(1+\eta^{2}\right)}
$$

where $\theta$ is the phase angle between the force and the displacement.

\section{Detailed model of a bolted joint with a viscoelastic layer}


A detailed bolted joint with a viscoelastic layer is shown in Fig. 3a. This represents the real joint shown in Fig. 4. The effective area of the viscoelastic layer in the whole joint is the area of the overlap less the area of the bolt hole. The thickness of the viscoelastic layer is $0.5 \mathrm{~mm}$. Ten node tetrahedral solid elements were used in the FE model shown in Fig. 3b. A $1.5 \mathrm{kN}$ preload was applied over the washer area, which can be seen in Fig. 3a. The left end of solid 3 was constrained by MPCs (multipoint constraints) so that the whole surface will have the same displacement. A force was applied on the independent node of the MPCs.. A quarter of a bolted joint was modeled according to symmetry conditions. The 3 planes of symmetry are i) the right hand edge of solid 1, ii) the bottom of solid 3 , and iii) the front surfaces of solids 1, 2, and 3. A similar mesh has been used in previous studies and has been found to give good results. A linear viscoelatic analysis was then undertaken.

The following stiffness matrix was used for the viscoelastic material:

$$
\left[K_{d d}\right]_{v}=\left\{\left[1+g_{R E F} T R(f)\right]+i\left[g+g_{R E F} T I(f)\right]\right\}\left[K_{1 d d}\right]_{v}
$$

where

$$
\begin{aligned}
& T R(f)=\frac{1}{\mathrm{~g}_{\mathrm{REF}}}\left[\frac{G^{\prime}(f)}{\mathrm{G}_{\mathrm{REF}}}-1\right] \\
& T I(f)=\frac{1}{\mathrm{~g}_{\mathrm{REF}}}\left[\frac{G^{\prime \prime}(f)}{\mathrm{G}_{\mathrm{REF}}}-\mathrm{g}\right]
\end{aligned}
$$

and $g$ is the global structural damping coefficient, which equals to twice the critical damping. The parameter $g_{R E F}$ is the reference structural damping coefficient of the viscoelastic material. It can be seen from (3) and (4) that $g_{R E F}$ is finally cancelled. It is included for convenience and the value it takes is not important. The parameter $G_{R E F}$ is the reference modulus of viscoelastic material and is also used only for the initialisation of matrix. $G^{\prime}(f)$ is the frequency dependent storage modulus and $G^{\prime \prime}(f)$ is the frequency dependent loss modulus of the viscoelatic material. These two parameters are used in the calculations. The matrix $\left[K_{d d}\right]_{v}$ is the stiffness matrix of the viscoelastic material computed on the basis of $G_{R E F}$. Futher details of equations (3) and (4) can be found in [13]. This stiffness matrix was incorporated into the dynamic equations of motion for the elements and then the equations were solved directly by numerical calculation.

The material of solid 1 and 3 is aluminium with an elastic modulus of $70000 \mathrm{MPa}$, a Poisson's ratio of 0.33 , and a density of $2800 \mathrm{~kg} / \mathrm{m}^{3}$. Many frequency response analyses have been undertaken. The results from a single example are discussed below for illustrative purposes:

At $1 \mathrm{~Hz}$ and an excitation force of $1000 \mathrm{~N}$ it was found that 


$$
\begin{aligned}
& x_{0}=0.5343 \mathrm{~mm} \\
& \theta=-33.7^{\circ} \\
& E=2 \pi F_{0} x_{0} \sin (\theta)=1.863 \mathrm{~J}
\end{aligned}
$$

where $x_{o}$ is the amplitude of the displacement at the free (left) end of the joint and $E$ is energy dissipated in one complete viscoelastic layer, This is why the factor 2 appears in both the finite element and the estimation formulae.

By using the following parameters

$$
\begin{aligned}
& F=2 \times 1000 \mathrm{~N}, \mathrm{~h}=0.5 \mathrm{~mm}, G=4148694 \mathrm{~Pa}, \eta=0.683 \\
& A=16 \times 25-\pi \times 3^{2} \mathrm{~mm}^{2}
\end{aligned}
$$

From Eq. (1) it can be found that

$$
\begin{aligned}
& x_{0}=0.00053550 \mathrm{~m} \\
& \theta=-34.333^{\circ}
\end{aligned}
$$

From Eq. (2) it can be found that

$$
E=1.898 \mathrm{~J}
$$

When the results from the FE model and the estimation formula are compared it is seen that the displacement error of the estimation formula was $0.22 \%$, the phase angle error was $1.9 \%$, and the error of dissipated energy was $1.9 \%$. It can be seen that using the estimation formula in the preliminary analyses is quite reasonable. A similar level of accuracy was found from other analyses with different loading parameters.

\section{B. Analytical models of bolted joints with viscoelastic layers}

In order to represent the bolted joints as spring-dashpots in the structural satellite model it is necessary to find the spring and dashpot parameters that represent the bolted joint response. The following equations can be obtained according to spring and dashpot mechanics in the Maxwell model.

$$
\begin{aligned}
& F_{1}=k_{m} x_{1} \\
& F_{2}=c_{m} \dot{x}_{2} \\
& F=F_{1}=F_{2} \\
& x=x_{1}+x_{2}
\end{aligned}
$$

From these equations, it can be shown that: 


$$
\frac{d x}{d t}=\frac{1}{k_{m}} \frac{d F}{d t}+\frac{F}{c_{m}}
$$

Assuming a harmonic excitation force, $F$

$$
F=F_{0} \cos \omega t
$$

Eq. (6) can be solved to give

$$
\begin{aligned}
x & =\frac{1}{k_{m}} F_{0} \cos \omega t+\frac{1}{c_{m} \omega} F_{0} \sin \omega t \\
& =F_{0} \sqrt{\frac{1}{k_{m}{ }^{2}}+\frac{1}{c_{m}{ }^{2} \omega^{2}}}\left(\frac{1}{k_{m} \sqrt{\frac{1}{k_{m}}+\frac{1}{c_{m}{ }^{2} \omega^{2}}}} \cos \omega t+\frac{1}{c_{m} \omega \sqrt{\frac{1}{k_{m}{ }^{2}}+\frac{1}{c_{m}{ }^{2} \omega^{2}}}} \sin \omega t\right) \\
& =x_{0} \cos (\omega t+\theta)
\end{aligned}
$$

where

$$
\begin{aligned}
& x_{0}=\frac{F_{0}}{k_{m} c_{m} \omega} \sqrt{c_{m}{ }^{2} \omega^{2}+k_{m}{ }^{2}}
\end{aligned}
$$

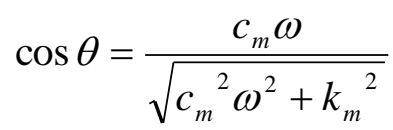

$$
\begin{aligned}
& \sin \theta=-\frac{k_{m}}{\sqrt{c_{m}{ }^{2} \omega^{2}+k_{m}{ }^{2}}}
\end{aligned}
$$

From above equations it can be shown that:

$$
\begin{aligned}
& k_{m}=\frac{F_{0}}{x_{0} \cos \theta} \\
& c_{m}=-\frac{F_{0}}{\omega x_{0} \sin \theta}
\end{aligned}
$$

By substituting the results from the detailed bolted joint model analysis (at $1 \mathrm{~Hz}$ ) into Eq. (10) it can be shown that

$$
\begin{aligned}
& k_{m}=4.499 \times 10^{6} \mathrm{~N} / \mathrm{m} \\
& c_{m}=1.07 \times 10^{6} \mathrm{~N} . \mathrm{s} / \mathrm{m}
\end{aligned}
$$

A spring dashpot FE model was created in Nastran. The parameters in Eq. (11) were used. The result was: 


$$
\begin{aligned}
& x=0.000534 \mathrm{~m} \\
& \theta=-33.7^{\circ} \\
& E=1.863 \mathrm{~J}(\text { from equation } 2 \mathrm{a})
\end{aligned}
$$

It can be seen that the spring dashpot model gave the same displacement and the same phase angle under the same excitation force as those given by the detailed joint model. Since the error between the estimation model and the detailed joint model was found to be small in section II.A.3, the former can be used to obtain the relationship between $A, G, \eta, h$, and $k_{m}, c_{m}$. This approach makes the modelling procedure simpler by removing the need for multiple bolted joint analyses.

Using the estimation formula (Eq. (1)) to obtain $\mathrm{k}$ and $\mathrm{c}$ through Eq. (10) gives:

$$
\begin{aligned}
& k_{m}=\frac{A\left(1+\eta^{2}\right) G}{h} \\
& c_{m}=\frac{A\left(1+\eta^{2}\right) G}{\omega h \eta}
\end{aligned}
$$

In the same way (also numerically tested in Nastran), the estimation formula of $k_{k}$ and $c_{k}$ for Kelvin model can be obtained:

$$
\begin{aligned}
& k_{k}=\frac{A G}{h} \\
& c_{k}=\frac{A G \eta}{h \omega}
\end{aligned}
$$

Equations (11) and (13) give appropriate equations to evaluate the equivalent Maxwell and Kelvin spring dashpot parameters from a specification of the basic viscoelastic material parameters. Either of these equations can be chosen for use in a spacecraft model. In the following section the Kelvin model is used.

\section{Developing a simple satellite model incorporating bolted joints with a viscoelstic layer}

\section{Satellite model}

The simple satellite model used is shown in Fig. 5. This satellite was composed of 7 honeycomb panels which were modelled with 2412 shell elements. On each bottom edge there were 18 joints and on each top edge there were 19 joints. So there were 74 joints and they were divided into 19 groups, numbers in each group being symmetrically located. For example the four node pairs at the top vertices of the satellite belong to group 10. The group numbers 
are shown in Fig. 5. The planes of symmetry were at the centre of the satellite and perpendicular to x and y axes. All groups had 4 node pairs except group 19 which was in the middle of the top edges and had only 2 node pairs. Each joint (or node pair) represented a bolted connection with a bolt on each of the two perpendicular faces Thus each pair of coincident nodes along the four edges was connected by three springs and three dashpots. These transmitted loads in the $\mathrm{x}, \mathrm{y}$, and $\mathrm{z}$ directions respectively. The coordinate system used is shown in the figure. The rotational degrees of freedom were constrained by MPCs.

\section{Modeling and results}

The shear modulus $G$ and the loss factor $\eta$ of the viscoelastic material in Table 1 were used in Eq. (13) along with geometry of the viscoelastic layer in the bolted joint (ie A and $\mathrm{h}$ in Eq. (13)). Several thicknesses of the viscoelastic layer were used to investigate the effect on the satellite response.

The width of the bolted joints was assumed to be $0.01 \mathrm{~m}$. The length of the satellite edge was $0.9 \mathrm{~m}$ and there were 19 bolts along each edge and the hole in the joint for the bolts was $5 \mathrm{~mm}$. Thus the shear area of each joint in Eq. (13) was calculated as

$$
A=0.9 \times 0.01 \div 19-\pi \times 2.5^{2} \times 10^{-6}=4.54 \times 10^{-4} \mathrm{~m}^{2}
$$

The frequency dependent spring dashpot parameters of the bolted joint with a layer thickness of $0.6 \mathrm{~mm}$ are shown in Table 2. Due to the orientation of the lines of joints the compliance in the global $Y$ direction is twice the compliance in the other two directions. Therefore, the stiffness and the damping coefficient of spring dashpot in the $Y$ direction were half those in $X$ and $Z$ directions.

Frequency response analyses were carried out with an excitation acceleration of $2 \mathrm{~g}$ and a global structural damping coefficient of 0.04 . These values are quite typical for the kind of satellite mentioned in this paper. It was found that the natural frequency was $34.76 \mathrm{~Hz}$. At this frequency the force-displacement response at each joint was obtained. The energy dissipated in all these joints was about $17.3 \mathrm{~J}$ which is $25 \%$ of the input energy, $70.2 \mathrm{~J}$. The damping ratio $\varsigma$ for this viscoelastic case was found by using half power bandwidth approach at point $\mathrm{N}$ to be about 0.024 .

Joints with $0.4 \mathrm{~mm}$ viscoelastic layers were also considered. The displacement of node $\mathrm{N}$ in the satellite model with $0 \mathrm{~mm}$ (none), $0.4 \mathrm{~mm}$, and $0.6 \mathrm{~mm}$ layers is shown in Fig. 6 . The von-Mises stress in element $M$ is shown in Fig. 7. The displacement decreased by about $9 \%$ and the stress decreased by about $10 \%$ from $0 \mathrm{~mm}$ to $0.6 \mathrm{~mm}$ cases. Due to this improved response, incorporating such damped bolted joints could result in a decrease in the satellite 
cost, for example, less material can be used due to the decrease of stress in structures. The thickness of the viscoelastic layers can be used to control the response of the satellite.

\section{Energy dissipated in a spacecraft structure incorporating non-linear viscoelastic layered bolted joints}

The focus of this section shifts to more complex, non-linear visco-elastic representation. Experimental work was undertaken to characterise bolted joints with damping layers operated in the non-linear domain. A simplified way of representing such non-linear behaviour of bolted joints was developed based on non-linear spring dashpots. Finally these were used to model the bolted joints in a satellite structure and a method of determining the damping of this system was developed and applied to the satellite structure.

\section{A. Experimental work}

In order to obtain a better understanding of real viscoelastic behaviour and to generate data to define the material property for modelling, a series of cyclic tests were carried out.

The joint tested was a double lap joint composed of two $25 \mathrm{mmx} 80 \mathrm{~mm} \times 6 \mathrm{~mm}$ aluminium bars with two $25 \mathrm{~mm} \times 34 \mathrm{~mm} \times 3 \mathrm{~mm}$ aluminium clamping plates. The clearance hole for the bolts had a diameter of $8 \mathrm{~mm}$ and the bolts were of $6 \mathrm{~mm}$ diameter. The clearance was sufficient to allow the viscoelastic layer to develop significant levels of strain. Thin layers of various viscoelastic materials were used between the clamping plates and the clamped bars. An assembled specimen is shown in Fig. 4.

An Instron 8511 test machine was used to undertake the experiments. When temperature is fixed, the main factors that could affect the non-linear behaviour of viscoelastic materials in the joints are frequency, strain level and preload. Sinusoidal dynamic tests were carried out to investigate these parameters. They included tests at various frequencies $(0.1 \mathrm{~Hz}, 1 \mathrm{~Hz}$, and $10 \mathrm{~Hz})$, with various cyclic displacement amplitudes (ranging between $0.1 \mathrm{~mm}$ and $0.8 \mathrm{~mm}$ ) and with preload torques varying between 6 N.m and 14 N.m.

The experimental material was a kind of silicone rubber named Versasil manufactured by Nusil. It cannot carry a significant load, but can be used to investigate typical viscoelasic behavior and validate the non-linear spring dashpot model that was developed. Before curing, the rubber is very flexible. It was rolled into a thin sheet and then 
cured in the oven at $100^{\circ} \mathrm{C}$ for about 1.5 hours. Following this it is like a normal rubber and can be cut into the shape needed for insertion into the joints.

A VersaSil 4050 gasket having a thickness of about $0.8 \mathrm{~mm}$ was used in the cyclic tests. At this thickness the maximum shear strain could be $125 \%$ due to the limitation of the joint clearance of $1.0 \mathrm{~mm}$. This did not make full use of the ductility of the material. Different frequencies and different amplitudes of displacement were applied. Fig. 8 shows two hysteresis loops. One is at $1 \mathrm{~Hz}$ and the other is at $10 \mathrm{~Hz}$. The amplitude of the displacement was $0.1 \mathrm{~mm}$ in both cases. The stiffness of the rubber increased with frequency as discussed in the literature.

More experiments were carried out at $1 \mathrm{~Hz}$ with different displacement amplitudes in extensometer control. The results are shown in Fig. 9. From Figs. 8 and 9, the dependence of properties on both strain and strain rate can be observed, but the dependence was dominated by the strain amplitude effects.

In practice the joints would be required to sustain a higher shear force in order to be used in load carrying structures. However, the deformation of the joint is limited for two reasons, a) the clearance in the joint is limited and b) the deformation has to meet design requirements. For a fixed deformation when the rubber is thinner, the strain is higher and hence sustains a higher level of stress. As the elongation of the VersaSil material was quite large, the thickness of the rubber could be reduced to enable it to bear more force for a given displacement. It was also assumed that if a primer was used, the rubber should bond to the substrates and thus sustain more shear loading. Thus a thin layer of VersaSil 4050 was used with a primer to see if the shear force could be increased. For the results in Fig. 10 the thickness of the rubber used was about $0.15 \mathrm{~mm}$. The test was controlled by extensometer at an amplitude $0.4 \mathrm{~mm}$ and a frequency $1 \mathrm{~Hz}$. The Mullins effect is clearly apparent in the data.

It can be seen from Fig. 10 that the shear force did increase compared with the data in Fig. 9, changing from about $200 \mathrm{~N}$ to $500 \mathrm{~N}$ in the stabilized state as the strain amplitude was increased. The stiffness of the rubber is still too small to be used for significant structural loading. However, the results can be used to correlate the numerical model that was developed

Some experiments were also undertaken to investigate the effect of the preload of the bolted joints on the resulting hysteresis loop. The results are shown in Fig. 11. Primer was used and the thickness of the rubber was about $0.5 \mathrm{~mm}$. A variety of preloading was applied through the use of $8 \mathrm{Nm}, 10 \mathrm{Nm}, 12 \mathrm{Nm}$, and $14 \mathrm{Nm}$ tightening torques. It appears that the effect of the preload was not very significant. The stiffness of the rubber increased slightly with an increase in the preload. 


\section{B. Modeling of bolted joints with viscoelastic layers}

It was necessary to incorporate the non-linear viscoelastic bolted joints into a satellite structure to investigate the global response of the satellite. The question is, how to achieve this? It can be seen from the literature review that much research has been carried out on the non-linear behavior of viscoelastic materials. Some good constitutive models have been developed. However, these models need to be included in a detailed joint model and it was not possible to include this level of detail in the global satellite model. Thus a simpler representation of the non-linear bolted joint response has been developed in this paper.

For a linear spring-dashpot model like the Kelvin model, which is composed of a spring and a dashpot in parallel, the force-displacement hysteresis loop is ellipse. Based on this, it may be conjectured that if the spring became nonlinear, the hysteresis loop may change and become more like the experimental data shown in Figs. 8-11. Further, if one can define the non-linear values of stiffness and damping at any point, it may be possible to derive an exact match. The spring-dashpot model used in this section is shown in Fig. 12. It was a non-linear spring (whose stiffness depends on the relative movement of the two nodes) and a linear dashpot (whose damping coefficient is a constant). It may be easier to control the shape using stiffness instead of damping (or both) because the stiffness is more directly related to the stiffness of the system. The value of the stiffness $k(\Delta)$ and the constant damping coefficient $c$ can be determined from the experimental results so that the system can replicate the observed behaviour of the joints.

Different parameters for the spring and dashpot were used to assess the effect of the parameters on the resulting loop shape. The force-deformation relationship was taken to be of the form

$$
F=a \Delta^{b}=k(\Delta) \Delta
$$

Different parameters (as shown in Table 3) were used for the systems. The results are shown in Fig. 13. It can be see from Fig. 13 that if a proper form of non-linear spring was chosen, it might be possible to replicate the data seen in the experiments. By observing the loops in the figures showing the experimental data it can be seen that at both ends of the loops the stiffness is bigger and in the middle the stiffness is smaller. A stiffness like that shown in Eq. (14) was used to fit the curve.

$$
k=a_{1} \times \Delta^{2}+a_{2} \times \Delta+a_{3}
$$


One set of the experimental data (the last loop from Fig. 10) was selected and used to find the corresponding stiffness of the spring so that the correlation between the spring-dashpot system is as close as possible to the experimental data. An optimization function in Matlab was used to find the best coefficients $a_{1}, a_{2}, a_{3}$ in Eq. (14) and the best damping coefficient constant c. The objective function used was

$$
\sum\left(f_{i}-F_{i}\right)^{2}
$$

where $f_{i}$ is the experimental force, $F_{i}$ is the numerically predicted force at the same displacement. The results are shown in Fig. 14.

The same optimization procedure was used to find the best stiffness and the best damping coefficient of a linear spring-dashpot system. This is also shown in Fig. 14. It can be seen that the curve from the non-linear spring dashpot system is much closer to the experimental data. Interestingly it was found the energy error (4.5\%) of the linear system was smaller than the nonlinear system $(5.7 \%)$. The energy error is calculated from:

$$
(e-E) / e
$$

where $e$ and $E$ are experimental energy and numerical energy separately.

This problem was resolved by defining another objective function including energy dissipated as well as force:

$$
\sum\left(f_{i}-F_{i}\right)^{2}+w|e-E|
$$

where $w$ is simply a weighting parameter used to balance force and energy errors. When w was large enough, the energy error can be quite small (1\%) for both linear and non-linear systems. To give a comprehensive evaluation of the systems the force error was also calculated, which is given as

$$
\sum\left|f_{i}-F_{i}\right| / \sum f_{i}
$$

Some results are shown in Table 4. It can be seen that if only the energy is of concern, both linear and non-linear systems can provide a good representation of the material. If the force and the energy are both of concern, the nonlinear system is a much better representation.

\section{Incorporating non-linear joints into the satellite model}

Such non-linear spring-dashpot joints need to be included in the global satellite model. In order to see if they can be used in an FE model of the satellite where there are many bolted joints and to investigate their effect on the 
behavior of the whole structure, the same simple satellite model shown in Fig. 4 was used. The non-linear joints were on the four edges at the top and bottom of the satellite, in the same positions as in the linear modeling, Fig. 4. They were also divided into the same 19 groups as the linear ones.

A non-linear material was defined, the behavior of which is shown in Fig. 15. The parameters were chosen by comparing with a linear model, to ensure that the stiffnesses and the energies dissipated will be similar. A stiffness $\mathrm{k}$ of $7 \times 10^{6} \mathrm{~N} / \mathrm{m}$ and a damping coefficient $\mathrm{c}$ of $4 \times 10^{4} \mathrm{~N} . \mathrm{s} / \mathrm{m}$ were chosen for the linear spring-dashpot model, which are close to the values used in simple satellite model in Section II when the thickness of the viscoelastic layer was $0.6 \mathrm{~mm}$ and the frequency was around $30 \mathrm{~Hz}$. After several numerical trials the following parameters were used:

$$
\begin{aligned}
& k=5 \times 10^{14} \Delta^{2}+2 \times 10^{6} \Delta(\mathrm{N} / \mathrm{m}) \\
& c=10000(\mathrm{~N} . \mathrm{s} / \mathrm{m})
\end{aligned}
$$

These parameters were used in the $\mathrm{y}$ direction at each joint. In the $\mathrm{x}$ and $\mathrm{z}$ directions linear spring-dashpots with stiffness $\mathrm{k}$ of $1.4 \times 10^{7} \mathrm{~N} / \mathrm{m}$ and damping coefficient $\mathrm{c}$ of $8 \times 10^{4}$ were used. This is because in these two directions the stiffnesses were twice as large (see Table 2) and the energy dissipated in them was quite small compared with the energy dissipated in the $Y$ direction.

It is difficult to find the natural frequency when using the non-linear model because the techniques available (for example frequency response analysis) do not consider the deformation dependency of the spring stiffness. From Eq. (15) and the deformation in Section II it can be seen that the stiffness of the springs lay between $2 \times 10^{6} \mathrm{~N} / \mathrm{m}$ and $7 \times 10^{6} \mathrm{~N} / \mathrm{m}$. Frequency response analyses with a structural damping coefficient of 0.04 were carried out with spring stiffness of $2 \times 10^{6} \mathrm{~N} / \mathrm{m}, 4 \times 10^{6} \mathrm{~N} / \mathrm{m}$, and $6 \times 10^{6} \mathrm{~N} / \mathrm{m}$. It was found the natural frequency was $34.18 \mathrm{~Hz}, 34.38 \mathrm{~Hz}$, and 34.52 $\mathrm{Hz}$ respectively. The aim of this section was to see the effect of the non-linearity on the structural response, so small differences in the natural frequency are not significant.

In fact there are ways to find the actual natural frequency. For example a fairly good result can be obtained by use of time consuming non-linear transient dynamic iterative calculations. It would also be appropriate to carry out analyses at several frequencies around the natural frequency to investigate the response of the model near the natural frequency. However, this was not the primary focus of concern of this work. In the following analyses a frequency of $34.35 \mathrm{~Hz}$ was chosen to see the effect of the non-linear spring dashpot model.

Non-linear transient analyses were carried out at $34.35 \mathrm{~Hz}$ on the simple satellite model. The structural damping coefficient was 0.04 and the excitation acceleration was $2 \mathrm{~g}$. The displacement of node N (Fig. 4) was obtained. It 
was $0.0009104 \mathrm{~m}$ when the excitation acceleration was $2 \mathrm{~m} / \mathrm{s}^{2}$ and was $0.009751 \mathrm{~m}$ when the excitation acceleration was $2 \mathrm{~g}$. If the model was linear the displacement of node $\mathrm{N}$ should be $0.008922 \mathrm{~m}$ at an excitation acceleration of $2 \mathrm{~g}$. The relative difference is $8.5 \%$. Assume that the non-linear model is an accurate model, then linear model will have an error of $8.5 \%$.

\section{Conclusions}

- This paper has outlined a possible methodology for representing bolted joints with visco-elastic layers in large structural models by using spring-dashpot models of varying degrees of complexity.

- Relationships have been derived which can be used to determine linear spring-dashpot properties in terms of the visco-elastic material properties and the geometry of the bolted joint.

- By undertaking dynamic FE analyses of a satellite structure that incorporates these simplified bolted joints there is evidence that it may be possible to dissipate a not insiginificant portion of the excitation energy in a typical bolted joint connections that include a visco-elastic layer.

- Experimental testing has shown that candidate materials for the visco-elastic layer may exhibit a significantly non-linear visco-elastic response.

- A non-linear spring dashpot model was developed that was a better fit to the experimental data than the linear model.

- By incorporating these models in the global satellite FE model it was shown that, for the configuration considered, the difference in predicted response between equivalent linear and non-linear models, for the same input, were of the order of $10 \%$ and thus the use of non-linear models are recommended.

\section{Appendix: List of notation}

$g=$ acceleration of gravity, global structural damping coefficient

$G^{*}=$ complex modulus of viscoelastic materials

$G \quad=\quad$ shear modulus of viscoelastic materials

$\eta=$ loss factor of viscoelastic materials

$F \quad=\quad$ excitation force

$A=$ shear area of a material element

$x=$ displacement

$h=$ thickness of a material element

$e, E=$ energy dissipated

$\theta=$ phase angle between force and displacement

$\left[K_{d d}\right]_{v}=$ stiffness matrix of viscoelastic materials

$g_{\text {REF }}=$ reference structural damping coefficient of viscoelastic materials 


$$
\begin{aligned}
G_{R E F} & =\text { reference modulus of viscoelastic materials } \\
G^{\prime}(f) & =\text { frequency dependent storage modulus of viscoelastic materials } \\
G^{\prime \prime}(f) & =\text { frequency dependent loss modulus of viscoelastic materials } \\
k & =\text { stiffness of spring } \\
c & =\text { damping coefficient of dashpot } \\
\omega & =\text { radian frequency }
\end{aligned}
$$

\section{References}

1 Crocombe A. D., Wang R., Richardson G., and Underwood C. I., "Estimating the Energy Dissipated in a Bolted Spacecraft at Resonance", Computers and Structures, Vol. 48, No. 5-6, 2006, pp. 340-350

2 Lkegami R., Johnson D. W., Walker W. J., and Beck C. J., "the Application of Viscoelastic Passive Damping to Satellite Equipment Support Structures”, Journal of Vibration, Acoustics, Stress and Reliability in Design, Vol. 107, 1985, pp. $367-374$.

3 Johnson C. D., and Kienholz D. A., "Prediction of Damping in Structures with Viscoelastic Materials", CSA Engineering Inc., 1983.

4 Mokeyev V., "a Generalized Complex Eigenvector Method for Dynamic Analysis of Heterogeneous Viscoelastic Structures”, International Journal for Numerical Methods in Engineering, Vol. 50, 2001, pp. 2271-2282.

5 Park S. W., "Analytical modelling of viscoelastic dampers for structural and vibration control”, International Journal of Solids and Structures, Vol. 38, 2001, pp. 8065-8092.

6 Ouis D., "Characterization of Polymers by Means of a Standard Viscoelastic Model and Fractional Derivate Calculus", International Journal of Polymeric Material, Vol. 53, 2004, pp. 633-644.

7 Kalgaonkar R. A., Nandi S., Tambe S. S., and Jog J. P., "Analysis of Viscoelastic Behaviour and Dynamic Mechanical Relaxation of Copolyester Based Layered Silicate Nanocomposites Using Havriliak-Negami Model", Journal of Polymer Science: Part B: Polymer Physics, Vol. 42, 2004, pp. 2657-2666.

8 Lee L.-H., Adhesive Bonding, Plenum Press, 1991.

9 Banks H. T., Pinter G A, Potter L. K., Gaitens M. J., and Yanyo L C, "Modeling of Nonlinear Hysteresis in Elastomers under Uniaxial Tension”, Journal of International Material Systems and Structures, Vol. 10, 1999, pp. 116-134.

10 Kitagawa M., Tatsuya M., and Tomohiko M., "Rate-dependent Nonlinear Constitutive Equation of Polypropylene", Journal of Polymer Science Part B-Polymer Physics, Vol. 27, No. 1, 1989, pp. 85-95.

11 Bergstrom J. S., and Boyce M. C., "Constitutive Modeling of the Large Strain Time-dependent Behavior of Elastomers", J. Mech. Phys. Solids, Vol. 46, No. 5, 1998, pp. 931-951.

12 Stahle C. V., and Staley J. A., "Application of Damping to Spacecraft Structures", National SAMPE Symposium and Exhibition (Proceedings), 1984, pp. 185-194. 
13 MSC.Nastran/Patran documentation, Macheal-Schwendler Corporation, 2005 
Table 1 Stiffness and damping properties of SMRD $100 \mathrm{~F} 90$ (at $25^{\circ} \mathrm{C}$ )

\begin{tabular}{cccc}
\hline \hline $\begin{array}{c}\text { Frequency } \\
(\mathrm{Hz})\end{array}$ & $\begin{array}{c}\text { Storage modulus } \\
\left(\times 10^{6} \mathrm{~Pa}\right)\end{array}$ & $\begin{array}{c}\text { Damping modulus } \\
\left(\times 10^{6} \mathrm{~Pa}\right)\end{array}$ & Loss factor \\
\hline 1 & 4.15 & 2.83 & 0.683 \\
1.778 & 5.09 & 4.17 & 0.820 \\
3.162 & 6.72 & 5.97 & 0.888 \\
5.623 & 8.20 & 7.57 & 0.924 \\
10 & 10 & 10 & 1 \\
17.78 & 13.7 & 14.9 & 1.08 \\
31.62 & 18.9 & 21.3 & 1.13 \\
56.23 & 25.9 & 29.2 & 1.13 \\
100 & 33.4 & 36.2 & 1.08 \\
\hline \hline
\end{tabular}

Table 2 Frequency dependent properties of spring dashpot (for a joint with $0.6 \mathrm{~mm}$ layer)

\begin{tabular}{ccccc}
\hline \hline$f(\mathrm{~Hz})$ & $\begin{array}{c}K_{x} K_{z} \\
\left(\times 10^{6} \mathrm{~N} / \mathrm{m}\right)\end{array}$ & $\begin{array}{c}C x C z \\
\left(\times 10^{4} \mathrm{~N} . \mathrm{s} / \mathrm{m}\right)\end{array}$ & $K_{\mathrm{y}}\left(\times 10^{6} \mathrm{~N} / \mathrm{m}\right)$ & $C_{y}\left(\times 10^{4} \mathrm{~N} . \mathrm{s} / \mathrm{m}\right)$ \\
\hline 1 & 3.16 & 34.3 & 1.58 & 17.2 \\
1.778 & 3.87 & 28.4 & 1.94 & 14.2 \\
3.162 & 5.11 & 22.9 & 2.56 & 11.4 \\
5.623 & 6.24 & 16.3 & 3.12 & 8.15 \\
10 & 7.61 & 12.1 & 3.80 & 6.05 \\
17.78 & 10.4 & 10.1 & 5.23 & 5.06 \\
31.62 & 14.4 & 8.14 & 7.18 & 4.07 \\
56.55 & 19.7 & 6.27 & 9.86 & 3.13 \\
100 & 25.4 & 4.38 & 12.7 & 2.19 \\
\hline \hline
\end{tabular}

Table 3 Parameters for different spring-dashpot system

\begin{tabular}{|c|c|c|c|c|c|c|}
\hline & \multicolumn{3}{|c|}{$\begin{array}{c}\text { Parameters of curves in } \\
\text { Fig. } 13 \mathrm{a}\end{array}$} & \multicolumn{3}{|c|}{$\begin{array}{c}\text { Parameters of curves in } \\
\text { Fig. } 13 \mathrm{~b}\end{array}$} \\
\hline & curve 1 & curve 2 & curve 3 & curve 1 & curve 2 & curve 3 \\
\hline $\begin{array}{l}\text { excitation } \\
\text { force }(\mathrm{N})\end{array}$ & 100 & 300 & 500 & 1000 & 3000 & 5000 \\
\hline$a\left(\mathrm{~N} / \mathrm{m}^{\mathrm{b}}\right)$ & & 10000 & & & 10000 & \\
\hline & & 3 & & & 0.6 & \\
\hline$c(\mathrm{Ns} / \mathrm{m})$ & & 10 & & & 100 & \\
\hline
\end{tabular}

Table 4 Comparison of linear and non-linear system

\begin{tabular}{ccccc}
\hline \hline \multirow{2}{*}{$w$} & \multicolumn{2}{c}{ linear } & \multicolumn{2}{c}{ non-linear } \\
\cline { 2 - 3 } & energy error & force error & energy error & force error \\
\hline 0 & $2.6 \%$ & $16.2 \%$ & $4.7 \%$ & $7.7 \%$ \\
10000 & $0.042 \%$ & $16.3 \%$ & $0.035 \%$ & $7.6 \%$ \\
\hline \hline
\end{tabular}




\section{List of figures}

Fig. 1 Viscoelastic properties for SMRD 100F90

Fig. 2 Simple model of viscoelastic material

Fig. 3 Detailed bolted joint with viscoelasic layer a) dimension of the joint and b) FE Model

Fig. 4 Bolted joint with viscoelastic layers

Fig. 5 Simple satellite model

Fig. 6 The variation of displacement of node $N$ with thickness of viscoelastic layer

Fig. 7 The von Mises stress of element $M$ with thickness of viscoelastic layer

Fig. 8 Hysteresis loops of a bolted joint with VersaSil 4050 at $1 \mathrm{~Hz}$ and $10 \mathrm{~Hz}$

Fig. 9 Hysteresis loops of a bolted joint with VersaSil $4050(1 \mathrm{~Hz})$

Fig.10 Hysteresis loops of a bolted joint with thin VersaSil 4050

Fig. 11 The effect of pre-load on the hysteresis loops

Fig. 12 Non-linear spring dashpot model

Fig. 13 Force displacement loops for spring-dashpot system (a) $F=10000 \Delta^{3}$ and (b) $F=10000 \Delta^{0.6}$

Fig. 14 The variation of experimental data with data from optimised spring dashpot system for VersaSil

Fig. 15 Comparison of the non-linear and the linear spring dashpot models 


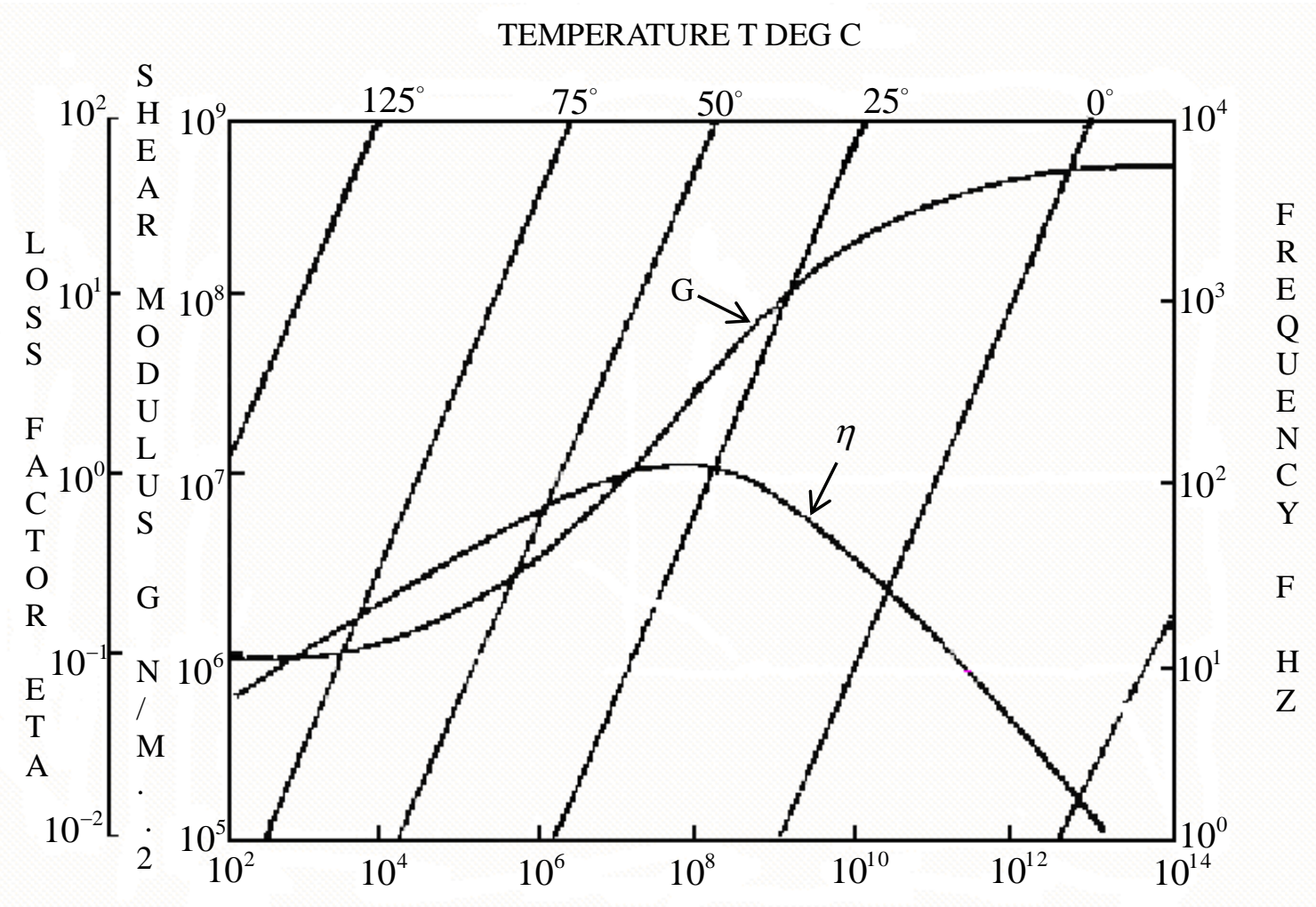

Fig. 1 Viscoelastic properties for SMRD $100 \mathrm{F90}{ }^{12}$

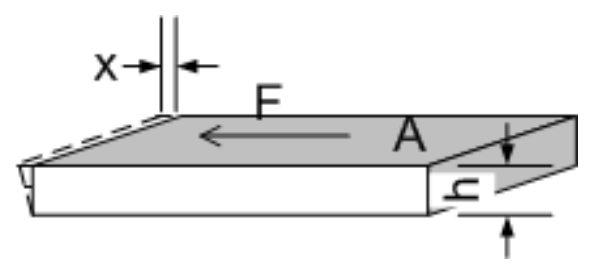

Fig. 2 Simple model of viscoelastic material

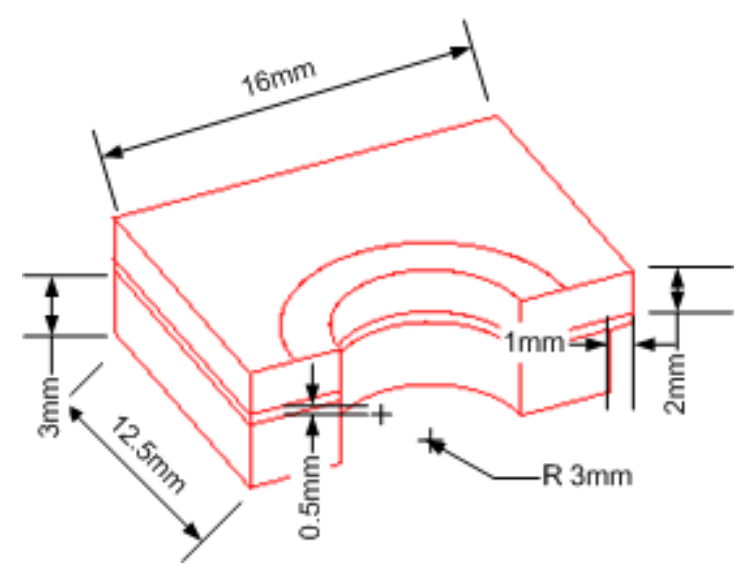

a)

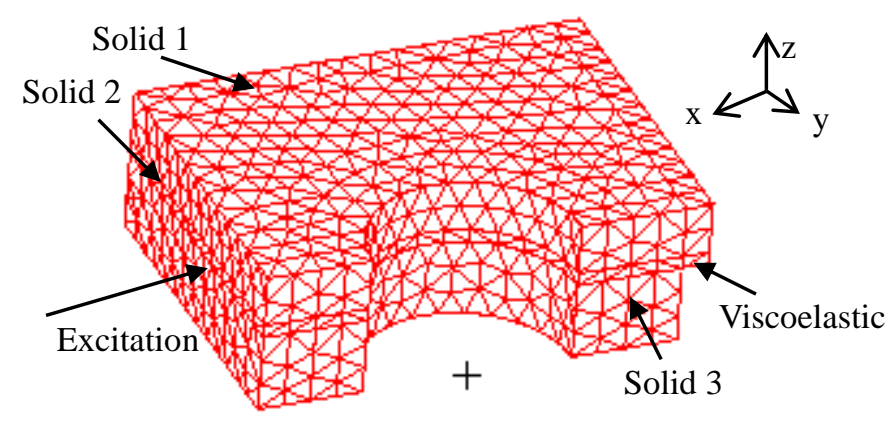

b) 
Fig. 3 Detailed bolted joint with viscoelasic layer a) dimension of the joint and b) FE

\section{Model}

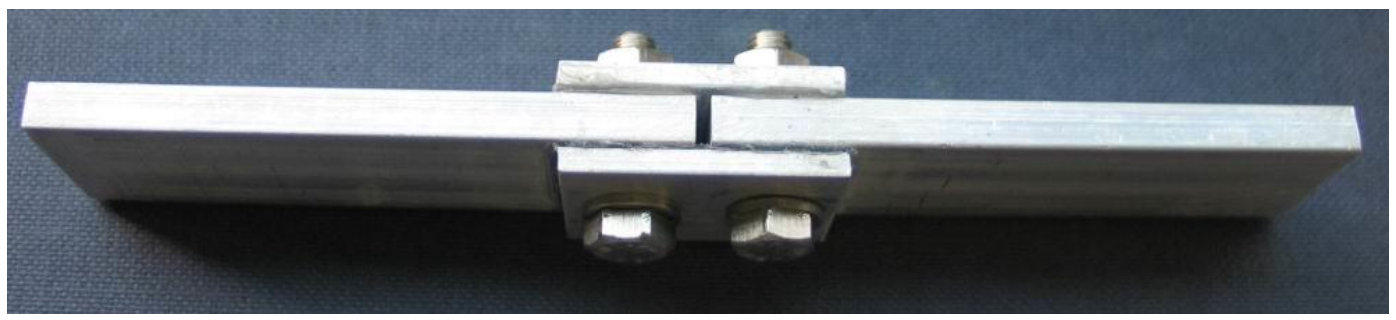

Fig. 4 Bolted joint with viscoelastic layers

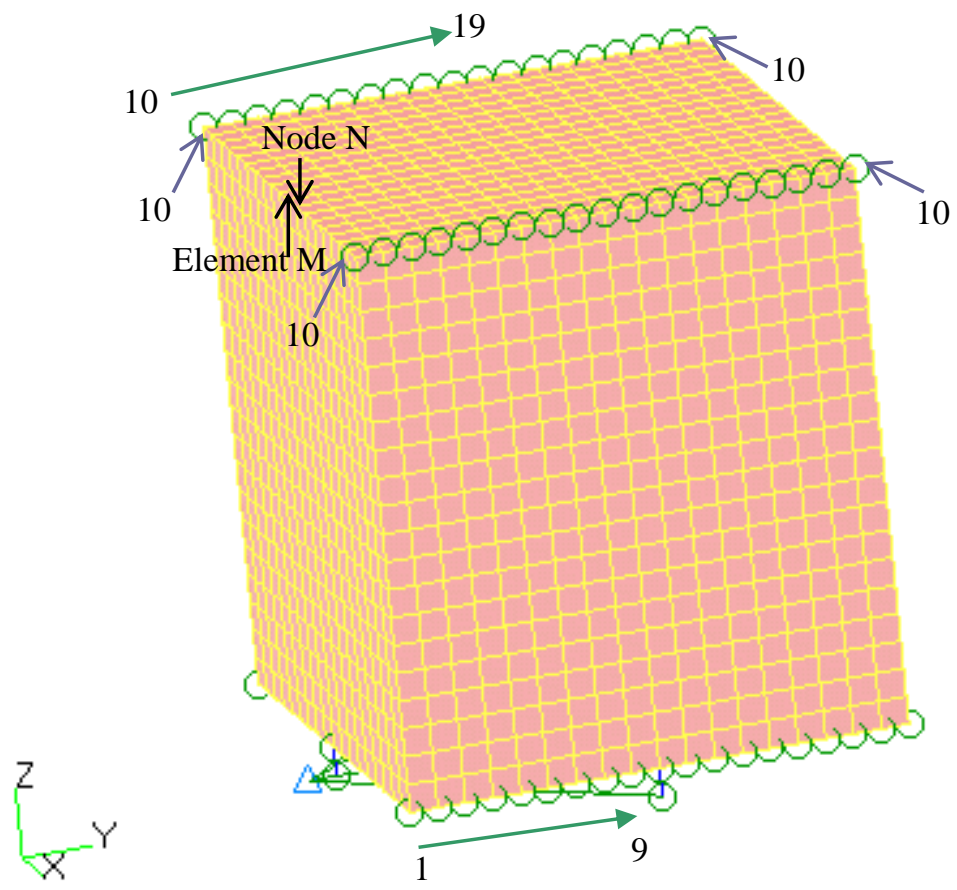


Fig. 5 Simple satellite model

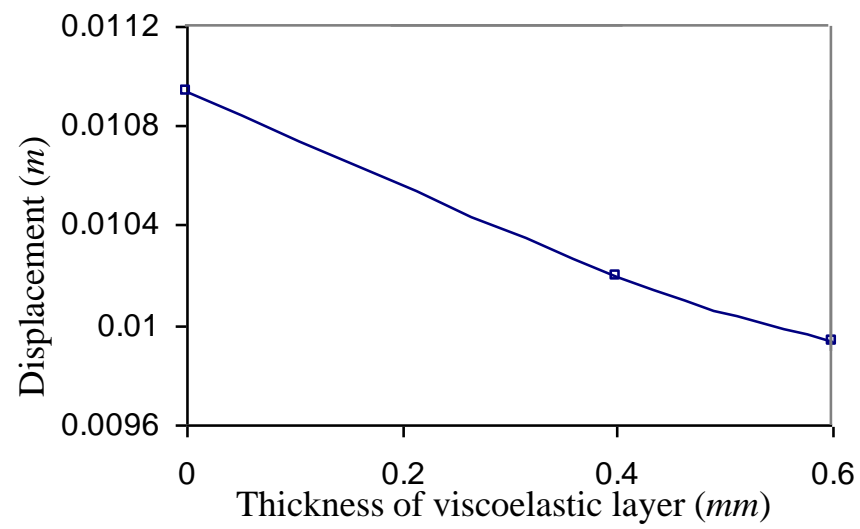

Fig. 6 The variation of displacement of node $\mathrm{N}$ with thickness of viscoelastic layer

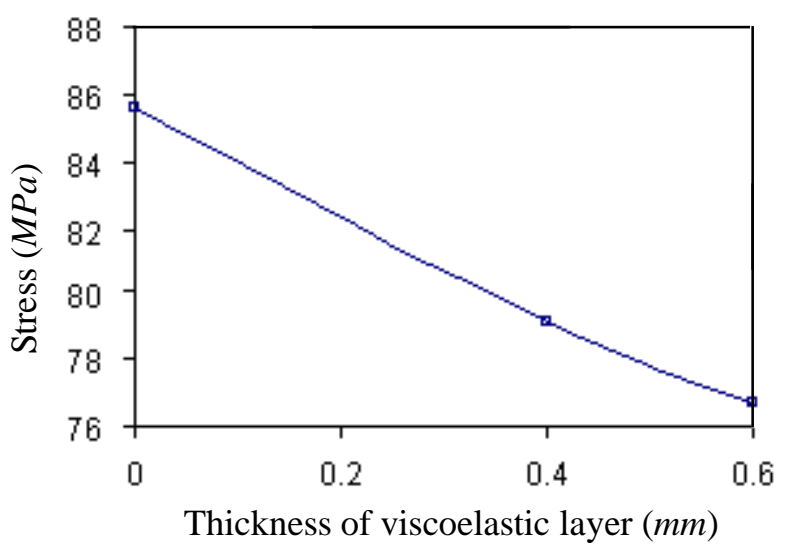

Fig. 7 The von Mises stress of element $M$ with thickness of viscoelastic layer 


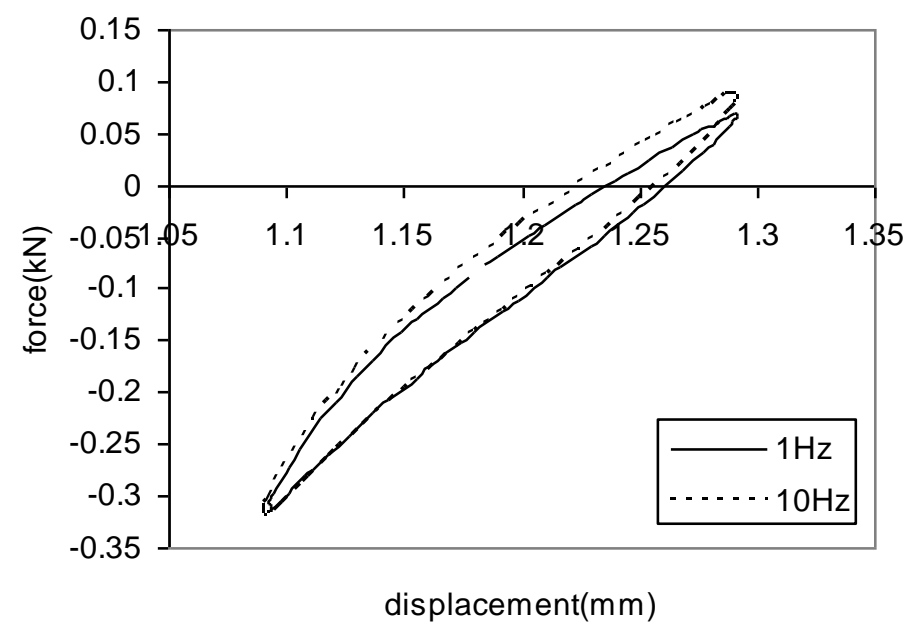

Fig. 8 Hysteresis loops of a bolted joint with VersaSil 4050 at $1 \mathrm{~Hz}$ and $10 \mathrm{~Hz}$

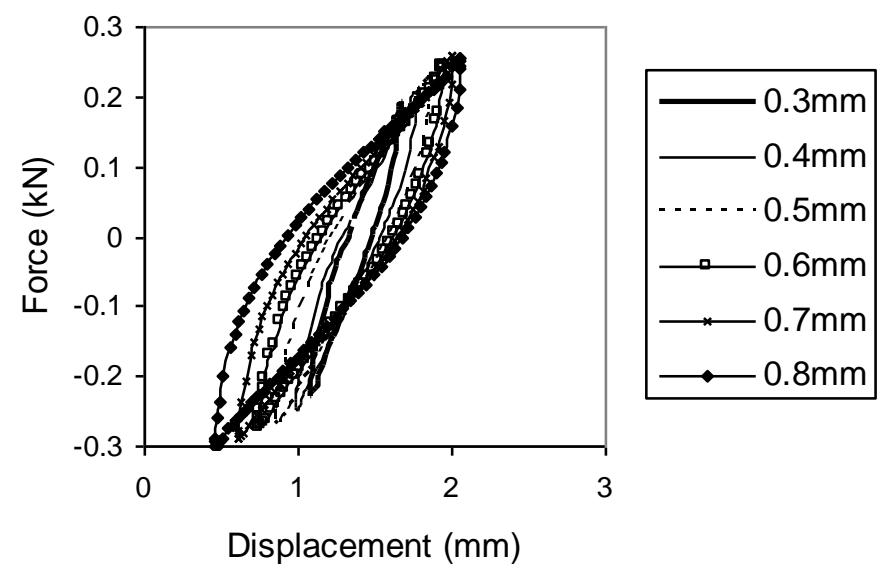

Fig. 9 Hysteresis loops of a bolted joint with VersaSil $4050(1 \mathrm{~Hz})$ 


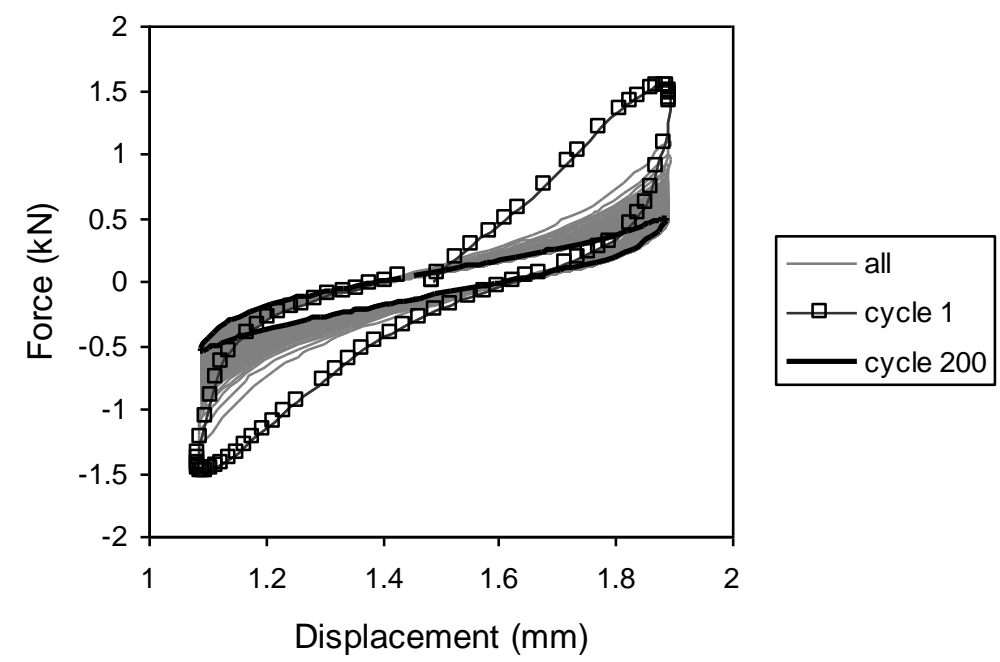

Fig. 10 Hysteresis loops of a bolted joint with thin VersaSil 4050

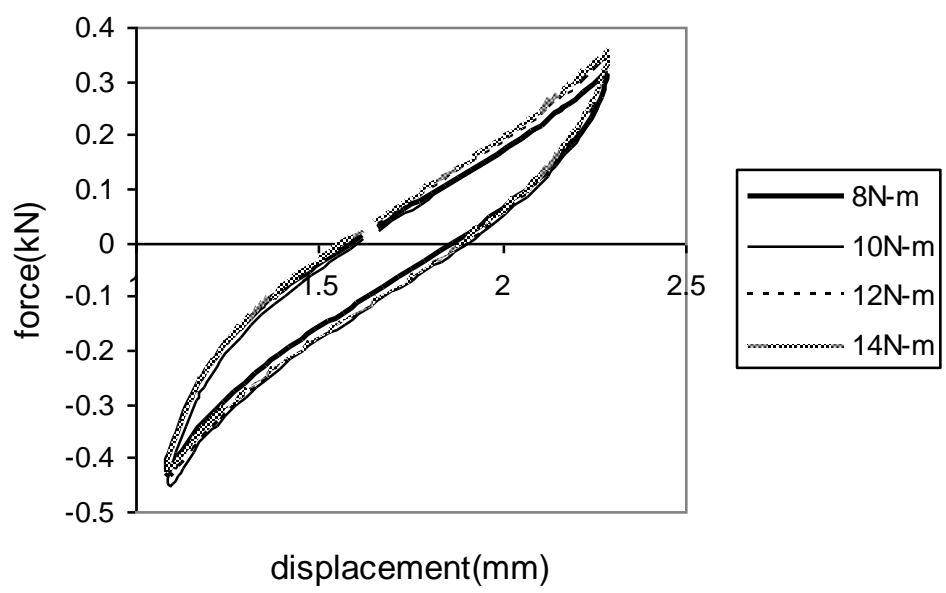

Fig.11 The effect of pre-load on the hysteresis loops

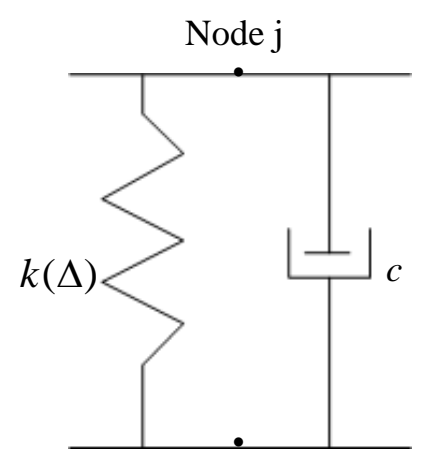

Node i 
Fig. 12 Non-linear spring dashpot model

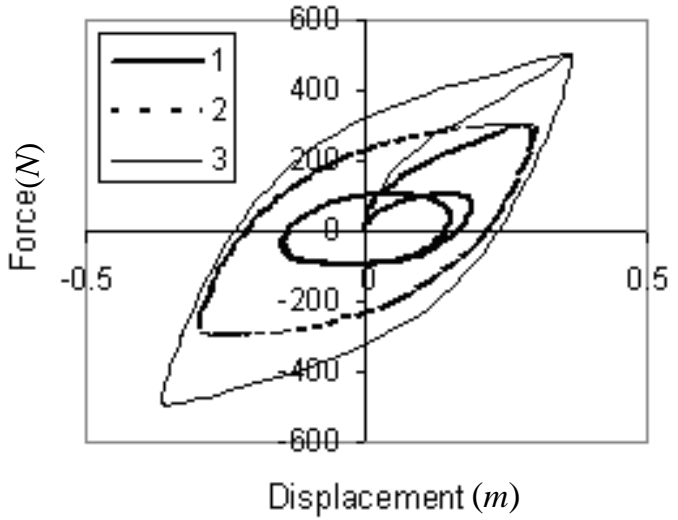

a)

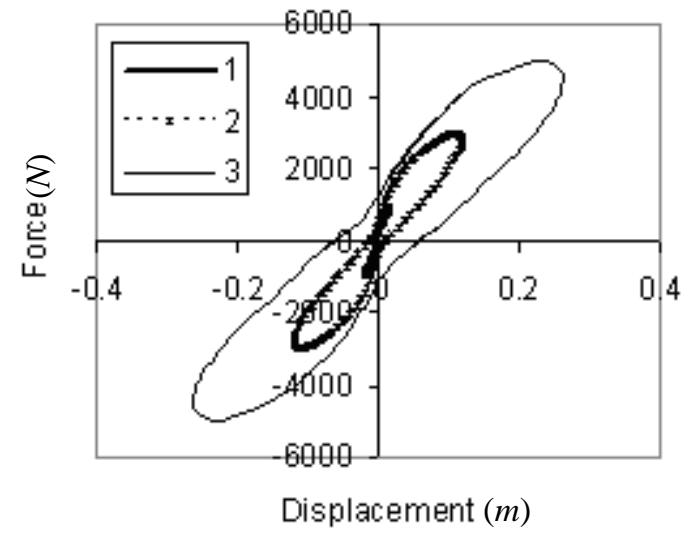

b)

Fig. 13 Force displacement loops for spring-dashpot system

(a) $\mathrm{F}=10000 \Delta^{3}$ and $(\mathrm{b}) \mathrm{F}=10000 \Delta^{0.6}$

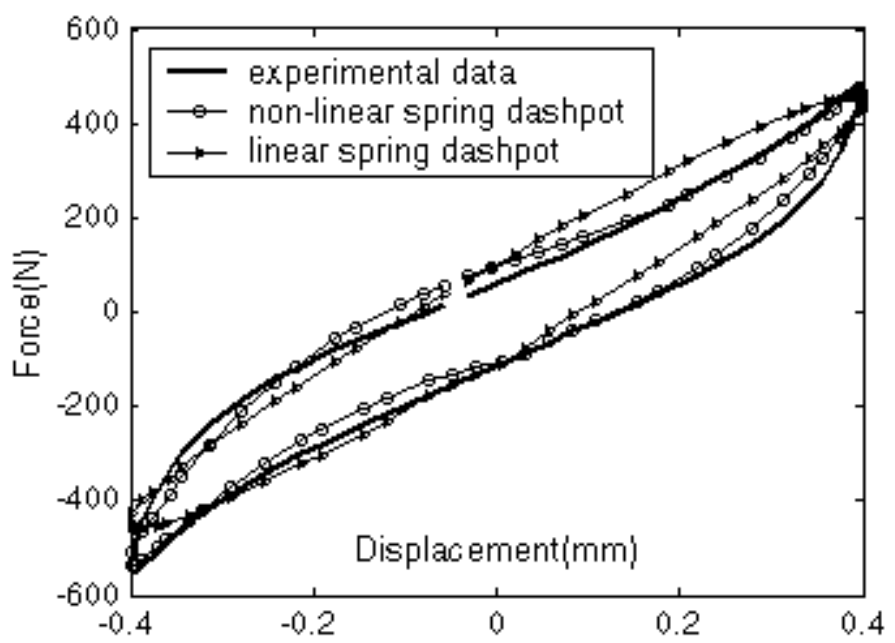

Fig. 14 The variation of experimental data with data from optimised spring dashpot system for VersaSil 


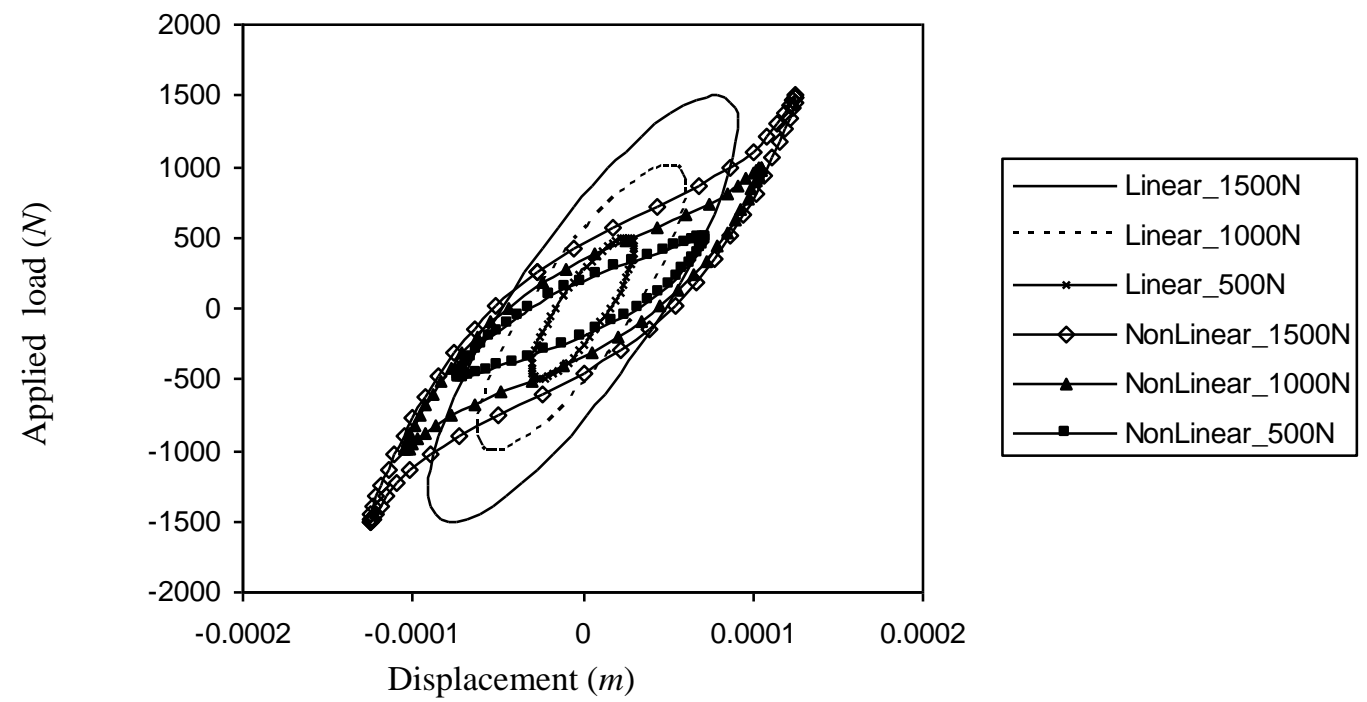

Fig. 15 Comparison of the non-linear and the linear spring dashpot models 